Educación Física y Ciencia, vol. 19, n 1, e025, junio 2017. ISSN 2314-2561

Universidad Nacional de La Plata.

Facultad de Humanidades y Ciencias de la Educación.

Departamento de Educación Física

\title{
Asociación entre aptitud cardiorrespiratoria y riesgo cardiovascular en el ingreso a la carrera de Educación Física
}

\author{
Association between cardiorespiratory fitness and cardiovascular \\ risk in the entrance to Physical Education course of study
}

\author{
Guillermo Pablo Maroni *; Horacio Martín Polo *; Marcelo Ghioldi **; \\ Eva Ybarra **; Martín Gustavo Farinola *
}

* ISEF N 2 "Federico W. Dickens", Argentina; ** Hospital Ramos Mejía, Argentina | guillermomaroni@gmail.com

\section{PALABRAS CLAVE}

\section{RESUMEN}

Factores de riesgo

Objetivo Estudiar la relación entre la aptitud cardiorrespiratoria y la agrupación de factores de riesgo cardiovascular en adultos jóvenes. Metodología Los sujetos fueron 278 varones y 151 mujeres de 18 a 25 años de edad ingresantes a la carrera de profesor de Educación Física en 2012 y 2013. El diseño fue transversal correlacional. La aptitud cardiorrespiratoria se valoró con la prueba de Course Navette y se comparó la agrupación de factores de riesgo en el grupo de mayor aptitud cardiorrespiratoria (cuartil superior) con el de menor aptitud (cuartil inferior). Los factores de riesgo fueron colesterol total, colesterol lipoproteína de alta densidad, glucemia, presión arterial sistólica y sumatoria de 5 pliegues cutáneos. Luego se dividió la muestra en tres: sin factores de riesgo, con uno o dos factores de riesgo y con tres o más. Se utilizó la prueba $\mathrm{Chi}^{2}$ para establecer la asociación entre la aptitud cardiorrespiratoria y la agrupación de factores de riesgo. Resultados Tanto en varones como en mujeres una baja aptitud cardiorrespiratoria se asoció significativamente con la agrupación de factores de riesgo $(\mathrm{p}<0,05)$. Conclusiones En esta muestra de jóvenes argentinos el perfil de factores de riesgo es más favorable cuanto mayor sea su aptitud cardiorrespiratoria.

\section{KEYWORDS}

Risk factors

Cardiorespiratory fitness

Young adults

\begin{abstract}
Objective To study the relationship between cardiorespiratory fitness and cardiovascular risk factors cluster in young adults. Methodology The subjects of this study were 278 males and 151 females between 18 and 25 years of age whose intention was to start the Physical Education course of study in 2012 and 2013. A cross-sectional correlational design was used. Cardiorespitary fitness was assessed with the Course Navette test and then the cardiovascular risk factors cluster in the group with the highest level of cardiorespiratory fitness (upper quartile) was compared to the group with lowest fitness. The cardiovascular risk factors studied were cholesterol, high density lipoprotein cholesterol, glycemia, systolic blood pressure, and sum of five skinfolds. The sample was divided in three subgroups: subjects without risk factors, with one or two risk factors, and with three o more. The Chi-square test was used to establish any association between cardiorespiratory fitness and risk factors cluster. Results Both men and women with a low cardiorespiratory fitness were significantly associated with clustering of risk factors $(\mathrm{p}<0.05)$. Conclusions In this sample of young Argentinean people, the risk factor profile is more favorable the higher their cardiorespiratory fitness is.
\end{abstract}




\section{Introducción}

Numerosos estudios han mostrado que altos niveles de aptitud cardiorrespiratoria se asocian con una baja prevalencia de factores de riesgo de enfermedades cardiovasculares y de morbilidad y mortalidad por enfermedades cardiovasculares (Blair, et al., 1989; Blair, et al., 1996; Ekelund, et al.,1988; Kokkinos, et al., 2008; Kokkinos, et al., 2010; Sandvick, et al.,1993).

Además, varios autores han investigado la asociación que existe entre aptitud cardiorrespiratoria y la agrupación (cluster) de factores de riesgo. LaMonte, Barlow, Jurca, Kampert y Church (2005) encontraron una asociación inversa significativa entre la aptitud cardiorrespiratoria (tertiles en un test máximo en cinta rodante) y agrupación de factores de riesgo (lípidos en ayunas, glucemia, presión arterial sistólica y circunferencia de cintura) en adultos de ambos sexos (44 \pm 9 años de edad) en un seguimiento longitudinal de 5,7 años.

Otros estudios fueron realizados en niños y jovenes de ambos sexos con una media de 9,6 y 15,5 años de edad respectivamente (Rizzo, Ruiz, Hurtig-Wennlöf, Ortega, Sjöstrom, 2005; Wedderkopp, Froberg, Hansen, Riddoch, Andersen, 2003). Estos estudios tuvieron metodologías similares aunque difirieron en la forma de categorizar la agrupación de factores de riesgo. En ambos estudios se estableció el nivel de aptitud cardiorrespiratoria a través de un test en cicloergometro, luego se los categorizó según el cuartil o el quintil en el que se ubicaron. Los factores de riesgo considerados fueron elcolesterol total, lipoproteína de alta densidad (colesterol HDL), glucemia, presión arterial sistolica (PAS) y la sumatoria de pliegues (4 o 5 pliegues, según el estudio). Luego, para Wedderkopp, et al. (2003) "tener un factor de riesgo" consistió en pertenecer al cuartil mas alto de ese factor de riesgo, mientras que en Rizzo et al. (2005) se utilizó el promedio del puntaje z de cada factor de riesgo. En ambos estudios, y con ambas técnicas de categorización de agrupación de factores de riesgo, los resultados fueron consistentes, un bajo nivel de aptitud cardiorrespiratoria se asoció con un mayor riesgo cardiovascular o metabólico.

En Argentina la enfermedad cardiovascular es la principal causa de muerte tanto en varones como en mujeres (Ministerio de Salud de la República Argentina, 2014), sin embargo la relación entre aptitud cardiorrespiratoria y los factores de riesgo cardiovascular no ha sido suficientemente estudiada. Por lo tanto en este trabajo nos proponemos investigar la relación entre la agrupación de factores de riesgo cardiovascular y la aptitud cardiorrespiratoria en una población de adultos jóvenes de la Ciudad Autónoma de Buenos Aires.

\section{Metodología}

\section{Participantes}

Los sujetos fueron ingresantes a la carrera de profesor de educación física de un instituto terciario público de la Ciudad Autónoma de Buenos Aires. La unidad de análisis fue el ingresante a la carrera de Educación Física en el Instituto Superior de Educación Física $\mathrm{N}^{\circ} 2$, de ambos sexos, de 18 a 25 años de edad y que cuente con la constancia de salud expedida por un profesional médico para la realización de actividad física. El estudio se realizó al inicio de los años lectivos 2012 y 2013 con la totalidad de ingresantes que cumplieron con los requisitos de la unidad de análisis y que decidieron participar del estudio voluntariamente. La muestra quedó conformada por 278 varones y 151 mujeres.

A cada participante se les administró una prueba de campo de aptitud cardiorrespiratoria, se les realizó antropometría y se les solicitó análisis de sangre de laboratorio dentro de un mismo mes.

\section{Prueba de aptitud cardiorrespiratoria}

Se utilizó la prueba de Course Navette de 20 metros (CN-20) con palieres de 1 minuto para valorar el nivel de aptitud cardiorrespiratoria en función del palier alcanzado por cada sujeto. Este es un test de campo, 
continuo, progresivo, máximo y que utiliza una señal sonora para marcar el ritmo. Este test cuenta con requisitos de calidad aceptables para la estimación del consumo máximo de oxígeno en sujetos jóvenes y adultos de ambos sexos (Léger, Mercier, Gadoury, Lambert, 1988; Léger y Gadoury, 1989).

El test fue administrado por profesores de educación física capacitados para la toma del mismo en un mismo gimnasio cubierto con suelo de cemento y utilizando un equipo de sonido con amplificador. Los sujetos se presentaron a un horario prefijado en grupos de 15 participantes. Realizaron una entrada calor con desplazamientos aeróbicos y elongación. Luego se les explicó el protocolo de la prueba y se dio comienzo a la misma. La prueba finalizó cuando los sujetos la abandonaron o a la segunda vez que los sujetos no llegaron a la siguiente marca junto con la señal sonora. Se registró el último palier completado.

Una vez administrada la prueba los participantes fueron agrupados por sexo y se dividió a cada grupo en cuartiles según el resultado obtenido. El cuartil 1corresponde al de menor nivel de aptitud cardiorrespiratoria y el cuartil 4 al de mayor nivel.

\section{Agrupación de factores de riesgo cardiovascular}

Los factores de riesgo cardiovascular utilizados fueron presión arterial sistólica (PAS), colesterol total, colesterol HDL, glucemia y sumatoria de 5 pliegues cutáneos: tríceps, subescapular, cresta ilíaca, abdominal, y supraespinal. Los pliegues fueron medidos según protocolos de la Sociedad Internacional para el Avance de la Cineantropometría (ISAK, por sus siglas en inglés) (Marfell-Jones, Olds, Stewart, Carter, 2006) por el mismo antropometrista quien contaba con veinte años de experiencia. La presión arterial fue medida con el participante sentado y el brazo a la altura del corazón. Los operadores encargados de la toma de la presión arterial fueron dos médicos de la División de Promoción y Protección de la Salud del Hospital Ramos Mejía de la Ciudad Autónoma de Buenos Aires quienes a su vez solicitaron los análisis de laboratorio. Al igual que la prueba cardiorrespiratoria, todos estos estudios fueron realizados durante el mismo mes (febrero) de 2012 y 2013 según el año de ingreso del participante.

Siguiendo a Wedderkopp, et al. (2003), para este estudio tener un factor de riesgo se definió como pertenecer al cuartil superior de ese factor de riesgo (el cuartil inferior para el caso del colesterol-HDL) para el sexo correspondiente. La agrupación de factores de riesgo se categorizó en una escala ordinal de tres valores: ausencia de factores de riesgo, presencia de 1 o 2 factores de riesgo, presencia de 3 o más factores de riesgo.

\section{Consideraciones éticas}

La información recogida fue anónima y confidencial. Los sujetos fueron informados del objetivo, contenido y características de las evaluaciones luego de lo cual firmaron un consentimiento informado para la realización de todas las pruebas.

\section{Tratamiento de datos}

Para el tratamiento descriptivo se calculó la media y el desvío estándar de las variables mencionadas a todo el conjunto y divido por sexo. Además, para caracterizar la muestra, se calculó la proporción de sujetos que obtuvieron valores saludables de cada variable según estándares internacionales para adultos (California Department of Education, 2015; Vorvick, 2014; Chen, 2014; Chen, 2015; Wisse, 2014). Luego se utilizó el test estadístico $\mathrm{Chi}^{2}$ para poder establecer la asociación entre la aptitud cardiorrespiratoria (cuartil más bajo vs cuartil más alto) y la agrupación de factores de riesgo de acuerdo a una escala de tres categorías (sin factores de riesgo, 1 o 2 factores de riesgo y 3 o más factores de riesgo) en cada sexo por separado. Se estableció un nivel de significancia de 0,05. El análisis estadístico se realizó con el software SPSS para Windows versión 11. 


\section{Resultados y discusión}

\section{Resultados}

En la tabla 1 se muestran las características de los sujetos y los valores que resultaron de corte en este trabajo para la aptitud cardiorrespiratoria y los diferentes factores de riesgo.

Tabla 1. Características de la muestra

\begin{tabular}{|c|c|c|c|c|c|c|}
\hline & \multicolumn{3}{|c|}{ Varones $(\mathrm{n}=278)$} & \multicolumn{3}{|c|}{ Mujeres $(\mathrm{n}=151)$} \\
\hline & Media & $\begin{array}{l}\text { Desvío } \\
\text { Estándar }\end{array}$ & $\begin{array}{l}\text { Proporción } \\
\text { Saludable }\end{array}$ & Media & $\begin{array}{l}\text { Desvío } \\
\text { Estándar }\end{array}$ & $\begin{array}{l}\text { Proporción } \\
\text { Saludable }\end{array}$ \\
\hline Edad (años) & 19,2 & 1,9 & $\begin{array}{c}\text { No } \\
\text { corresponde }\end{array}$ & 18,8 & 1,6 & $\begin{array}{c}\text { No } \\
\text { corresponde }\end{array}$ \\
\hline $\begin{array}{l}\text { Course } \\
\text { Navette de 20 } \\
\text { m (palieres) }\end{array}$ & 9,8 & 1,6 & $93 \%^{\mathrm{a}}$ & 6,1 & 1,4 & $66 \%^{\mathrm{a}}$ \\
\hline IMC $\left(\mathrm{kg} / \mathrm{m}^{2}\right)$ & 23,0 & 2,4 & $80 \%{ }^{\mathrm{b}}$ & 22,5 & 2,8 & $70 \%^{\mathrm{b}}$ \\
\hline $\begin{array}{l}\text { Colesterol } \\
\text { Total }(\mathrm{mg} / \mathrm{dL})\end{array}$ & 155,9 & 27,6 & $94 \%^{\mathrm{c}}$ & 163,3 & 30,1 & $89 \%^{\mathrm{c}}$ \\
\hline $\begin{array}{l}\text { Colesterol } \\
\text { HDL }(\mathrm{mg} / \mathrm{dL})\end{array}$ & 48,8 & 10,1 & $82 \%{ }^{\mathrm{c}}$ & 55,7 & 14,0 & $91 \%^{\mathrm{c}}$ \\
\hline PAS (mmHg) & 114,1 & 11,3 & $82 \%{ }^{\mathrm{d}}$ & 106,4 & 10,1 & $97 \%^{d}$ \\
\hline $\begin{array}{l}\text { Glucemia } \\
\text { (mg/dL) }\end{array}$ & 86,4 & 9,2 & $94 \%{ }^{\mathrm{e}}$ & 82,2 & 10,1 & $98 \%{ }^{\mathrm{e}}$ \\
\hline $\begin{array}{l}\text { Sumatoria de } \\
5 \text { Pliegues } \\
(\mathrm{mm})\end{array}$ & 60,0 & 25,2 & No disponible & 86,7 & 25,1 & No disponible \\
\hline
\end{tabular}

IMC: Índice de Masa Corporal.PAS: Presión Arterial Sistólica. Valores saludables: ${ }^{a}$ Varones: $\geq 8$ palieres; Mujeres: $\geq 6$ palieres (tomado de California Department of Education, 2015). ${ }^{\mathrm{b}} 18,5$ a 24,9 kg/m² (tomado de Vorvick, 2014). ${ }^{\mathrm{c} C o l e s t e r o l ~ t o t a l: ~} 180$ a $200 \mathrm{mg} / \mathrm{dL}$ o menos; Colesterol HDL: 40 a $60 \mathrm{mg} / \mathrm{dL}$ (tomados de Chen, 2014). ${ }^{\mathrm{d}}>120 \mathrm{mmHg}$ (tomado de Chen, 2015). ${ }^{\mathrm{e}}$ en ayunas 70 a $100 \mathrm{mg} / \mathrm{dL}$ (tomado de Wisse, 2014).

La tabla 2 muestra los puntos de corte que se utilizaron en este trabajo, adaptando la metodología de Wedderkopp, et al. (2003), para definir la agrupación de factores de riesgo. También se muestra la cantidad de palieres que resultaron ser los percentiles 25 y 75 en la prueba CN-20 en ambos sexos y así poder identificar a los sujetos de baja aptitud cardiorrespiratoria (primer cuartil) y a los de elevada aptitud (cuarto cuartil).

Tabla 2. Puntos de corte para los factores de riesgo y la aptitud cardiorrespiratoria utilizados en este trabajo*

\begin{tabular}{|c|c|c|}
\hline & Varones & Mujeres \\
\hline \multicolumn{3}{|l|}{ Factor de riesgo } \\
\hline Colesterol Total (mg/dL) & $\geq 176$ & $\geq 179$ \\
\hline Presión Arterial Sistólica (mmHg) & $\geq 120$ & $\geq 110$ \\
\hline Colesterol HDL (mg/dL) & $\leq 42$ & $\leq 45$ \\
\hline Glucemia (mg/dL) & $\geq 93$ & $\geq 88$ \\
\hline Sumatoria de 5 Pliegues (mm) & $\geq 71$ & $\geq 104$ \\
\hline \multicolumn{3}{|c|}{ Prueba cardiorrespiratoria (Course Navette de $20 \mathrm{~m}$ ) } \\
\hline
\end{tabular}




\begin{tabular}{|l|l|l|}
\hline Primer cuartil (palieres) & $\leq 9$ & $\leq 5$ \\
\hline Cuarto cuartil (palieres) & $\geq 11$ & $\geq 7$ \\
\hline
\end{tabular}

* ver Metodología para consultar la técnica utilizada para establecer estos valores.

En la tabla 3 se muestra la cantidad de sujetos de cada cuartil de aptitud cardiorrespiratoria en las diferentes categorías de agrupación de factores de riesgo cardiovascular, encontrándose una asociación significativa entre ambas variables en ambos sexos. En ambos sexos ocurrió que a medida que aumenta la agrupación de factores de riesgo disminuye la cantidad relativa de sujetos que cuentan con elevada aptitud cardiorrespiratoria (4to cuartil) y lo contrario sucede con los sujetos de bajo nivel de dicha capacidad (1er cuartil).

Tabla 3. Relación entre agrupación factores de riesgo y prueba de Course Navette 20 m (aptitud cardiorrespiratoria).

\begin{tabular}{|c|c|c|c|c|c|c|}
\hline & \multicolumn{4}{|c|}{ Course Navette 20 m } \\
\hline & & & \multicolumn{2}{|c|}{ Varones } & \multicolumn{2}{|c|}{ Mujeres } \\
\hline & & & $\begin{array}{c}\text { 1er Cuarti } \\
(n=97)\end{array}$ & $\begin{array}{l}\text { 4to Cuartil* } \\
\qquad(\mathrm{n}=91)\end{array}$ & $\begin{array}{c}\text { 1er Cuartil } \\
(\mathrm{n}=46)\end{array}$ & $\begin{array}{c}\text { 4to Cuartil** } \\
(\mathrm{n}=52)\end{array}$ \\
\hline \multirow{6}{*}{$\begin{array}{l}\text { Agrupación } \\
\text { de factores } \\
\text { de riesgo }\end{array}$} & \multirow{2}{*}{$\begin{array}{l}\text { Sin factores } \\
\text { de riesgo }\end{array}$} & $\mathrm{n}$ & 18 & 36 & 1 & 3 \\
\hline & & $\%$ & $33,3 \%$ & $66,7 \%$ & $25,0 \%$ & $75,0 \%$ \\
\hline & \multirow{2}{*}{$\begin{array}{l}1 \text { o } 2 \\
\text { factores de } \\
\text { riesgo }\end{array}$} & $\mathrm{n}$ & 58 & 45 & 30 & 44 \\
\hline & & $\%$ & $56,3 \%$ & $43,7 \%$ & $40,5 \%$ & $59,5 \%$ \\
\hline & \multirow{2}{*}{$\begin{array}{l}3 \text { o más } \\
\text { factores de } \\
\text { riesgo }\end{array}$} & $\mathrm{n}$ & 21 & 10 & 15 & 5 \\
\hline & & $\%$ & $67,7 \%$ & $32,3 \%$ & $73,7 \%$ & $26,3 \%$ \\
\hline
\end{tabular}

*Diferencias significativas con el 1er cuartil $\left(\mathrm{Chi}^{2} \mathrm{p} 0,003\right)$. **Diferencias significativas con el 1er cuartil (Chi ${ }^{2}$ p 0,024).

\section{Discusión}

La asociación entre aptitud cardiorrespiratoria y factores de riesgo cardiovascular se encuentra bien establecida internacionalmente. En Argentina, a pesar de que la enfermedad cardiovascular es la principal causa de mortalidad en adultos de ambos sexos, este tema no se encontraba estudiado. Nuestros resultados son consistentes con los antecedentes internacionales, tanto de jóvenes Rizzo, et al. (2005), Wedderkropp, et al. (2003) como de adultos LaMonte, et al. (2005), los cuales sugieren una asociación inversa entre la acumulación de factores de riesgo cardiovascular y la aptitud cardiorrespiratoria.

Una limitación de este estudio fue no haber utilizado puntos de corte de cada factor de riesgo para adultos (como por ejemplo en LaMonte, et al., 2005) en una muestra de sujetos que sí son adultos. Sin embargo esto lo creemos justificado por dos razones. Primero, y si bien los sujetos de este estudio fueron adultos, su edad estuvo comprendida entre los 18 y los 25 años, es decir muy cercana a la adolescencia en la cual sí se han utilizado los puntos de corte y la técnica de agrupación de factores de riesgo utilizada aquí. Y segundo, nuestra muestra (ingresantes a la carrera de profesor de Educación Física) suele contar con niveles de actividad física elevados (Farinola, Polo, La Valle, Arcuri, 2009) y no suele contar con factores de riesgo presentes según los estándares de salud para adultos (Tabla 1). Por lo tanto utilizar estos estándares en esta población no permitiría medir la asociación porque el grupo de sujetos con factores de riesgo agrupados es 
escaso o nulo. De todas formas la interpretación de nuestros resultados debe hacerse con cautela debido a esta decisión metodológica.

No obstante este perfil favorable de salud (según los estándares para adultos) y de actividad física de nuestra muestra, la asociación inversa entre aptitud cardiorrespiratoria y agrupación de factores de riesgo cardiovascular fue estadísticamente significativa, lo que sugiere que esta capacidad física es un marcador considerable de salud. A la misma conclusión llegaron Kokkinos, et al. (2010) quienes encontraron una asociación gradual y significativa entre aptitud cardiorrespiratoria y mortalidad y que aquellos sujetos que habían incrementado su aptitud redujeron su riesgo de mortalidad.

En este trabajo, además de la asociación significativa, también se observó una gradualidad y en ambos sexos (tabla 2). La mayoría de los sujetos sin factores de riesgo obtuvieron altos resultados en la prueba cardiorrespiratoria, y a medida que aumentó la presencia de factores de riesgo, el porcentaje de sujetos con buenos resultados en la prueba cardiorrespiratoria disminuyó, y esto ocurrió en ambos sexos. Esta gradualidad le da más fuerza a la idea de que la aptitud cardiorrespiratoria es un marcador fuerte de salud.

Creemos que este estudio es de relevancia debido a que es uno de los primeros, sino el primero, en ofrecer evidencia a favor de la asociación entre estas dos variables en Argentina. Sin embargo reconocemos que es un tema que merece seguir estudiándose en muestras que incluyan una mayor cantidad y heterogeneidad de sujetos y combinando con otras metodologías.

\section{Conclusiones}

Al igual que lo hallado internacionalmente, en esta muestra de jóvenes argentinos el perfil de factores de riesgo cardiovascular fue más favorable cuanto mayor fue su aptitud cardiorrespiratoria. Se sugiere continuar la investigación local en este tema ampliando las muestras y variando las metodologías.

\section{Referencias Bibliográficas}

Blair S.N., Kampert J., Kohl H.W. III, Barlow C., Macera C., Paffenbarger R.S. Jr, et al. (1996). Influences of cardiorespiratory fitness and other precursors on cardiovascular disease and all-Cause mortality in men and women. JAMA, 276(3), 205-210.

Blair S.N., Kohl H.W. III, Paffenbarger R.S., Clark D., Cooper K., Gibbons L. W. (1989). Physical fitness and all-causes mortality: A prospective study of healthy men and women. JAMA, 262(17), 2395-2401.

California Department of Education. FITNESSGRAM performance standards. (2015). Recuperado de http://www.cde.ca.gov/ta/tg/pf/documents/pft15hfzstd.pdf . Acceso el 01 de enero de 2015.

Chen M. (2014). Exámenes de colesterol y sus resultados. MedlinePlus. Biblioteca Nacional de Medicina de los Estados Unidos. Institutos Nacionales de Salud, Estados Unidos. Recuperado de https://www.nlm.nih.gov/medlineplus/spanish/ency/patientinstructions/000386.htm . Acceso el 01 de enero de 2015.

Chen M. (2015). Hipertensión arterial. MedlinePlus. Biblioteca Nacional de Medicina de los Estados Unidos. Institutos Nacionales de Salud, Estados Unidos. Recuperado de https://www.nlm.nih.gov/medlineplus/spanish/ency/article/000468.htm. Acceso el 01 de mayo de 2015.

Ekelund L.G., Haskell W.L., Johnson J., Whaley F., Criqui M., Sheps D.S. (1988). Physical fitness as a predictor of cardiovascular mortality in asymptomatic North American Men: The lipid research clinics mortality follow-up study. $N$ Engl J Med, 319(21), 1379-1384. 
Farinola MG, Polo M, La Valle L, Arcuri C. Niveles de actividad física en alumnos/as del Instituto Superior de Educación Física № 2 Federico W. Dickens. Revista electrónica de Ciencias Aplicadas al Deporte, 2009; 2(5). https://www.romerobrest.edu.ar/ojs/index.php/ReCAD/article/view/87.

Kokkinos P., Myers J., Faselis C., Panagiotakos D.B., Doumas M., Pittaras A., et al. (2010). Exercise capacity and mortality in older men a 20-year follow-up study. Circulation, 122(8), 790-797.

Kokkinos P., Myers J., Kokkinos J.P., Pittaras A., Narayan P., Manolis A., et al. (2008). Exercise Capacity and mortality in black and white men. Circulation, 117(5), 614-622.

LaMonte M., Barlow C., Jurca R., Kampert J., Church T. (2005). Cardiorespiratory fitness is inversely associated with the incidense of metabolic syndrome: A perspective study of men and women. Circulation, 112(4), 505-512.

Léger L., Gadoury C. (1989). Validity of the $20 \mathrm{~m}$ shuttle run test with 1 min stages to predict $\mathrm{VO}_{2} \max$ in adults. Can J Sport Sci, 14(1), 21-26.

Léger L., Mercier D., Gadoury C., Lambert J. (1988). The multistage 20 metre shuttle run test for aerobics fitness. Journal of Sports Sciences, 6(2), 93-101.

Marfell-Jones M., Olds T., Stewart A., Carter L. (2006). Estándares Internacionales para Mediciones Antropométricas. Recuperado de http://www.nutrisys-py.com/descarga/img/-Estandares\%20internacionales \%20para\%20Mediciones\%20Antropometricas.pdf. Acceso el 01 de enero de 2015.

Ministerio de Salud de la República Argentina. (2014). Indicadores Básicos Argentina 2014. Recuperado de http://www.deis.msal.gov.ar/publicaciones/archivos/indicadores 2014.pdf. Acceso el 01 junio de 2015.

Rizzo N.S., Ruiz J.R., Hurtig-Wennlöf A., Ortega F.B., Sjöstrom M. (2005). Relationship of physical activity, fitness and fatness with clustered metabolic risk in children and adolescents: The European youth heart study. J Pediatr, 150(4), 388-393.

Sandvick L., Erikssen J., Thaulow E., Erikssen G., Mundal R., Rodahl K. (1993). Physical fitness as a predictor of mortality among healthy, middle-aged Norwegian men. $N$ Engl J Med, 328(8), 533-537.

Vorvick L. (2014). Índice de masa corporal. MedlinePlus. Biblioteca Nacional de Medicina de los Estados Unidos. Institutos Nacionales de Salud, Estados Unidos. Recuperado de https://www.nlm.nih.gov/medlineplus/spanish/ency/article/007196.htm. Acceso el 01 de enero de 2015.

Wedderkopp N., Froberg K., Hansen H., Riddoch C., Andersen L. (2003). Cardiovascular risk factors cluster in children and adolescents with low physical fitness: The European youth heart study. Pediatric Exercise Science, 15(4), 419-427.

Wisse B. (2014). Examen de glucemia. MedlinePlus. Biblioteca Nacional de Medicina de los Estados Unidos. Institutos Nacionales de Salud, Estados Unidos. Recuperado de https://www.nlm.nih.gov/medlineplus/spanish/ency/article/003482.htm. Acceso el 01 de enero de 2015. 